\title{
Coágulos intraluminales en postoperatorio de una hepaticoyeyunostomía: rara complicación para un rápido actuar
}

Postoperative intraluminal blood clots after a hepaticojejunostomy: rare, fast-action complication

Coágulos intraluminais no pós-operatório de uma hepático-jejunostomia: uma complicação rara para uma ação rápida

Juan Carlos Marín Marmolejo, MD., Esp. ${ }^{1}$ (D), Luis Antonio Sarmiento, Est. ${ }^{2}$ (D), Juan David Martínez, Est. ${ }^{3}$ (D)

1. Médico, Especialista en Cirugía General. Cirujano coloproctólogo SES Hospital de Caldas, Colombia.

2. Médico Interno, Coordinador semillero de cirugía e investigación (SECIN), Universidad de Caldas. Colombia.

3. Médico Interno, Universidad de Caldas. Colombia.

Correspondencia. Luis Antonio Sarmiento. Carrera 27\# 46-66 apartamento 1 Barrio Lleras Manizales, Caldas, Colombia. Email. luissarmiento2407@gmail.com

\section{INFORMACIÓN DEL ARTÍCULO:}

Artículo recibido: 11 de enero 2020

Artículo aceptado: 25 de mayo 2020

DOI: https://doi.org/10.29375/01237047.3823

Cómo citar: Marín-Marmolejo JC, Sarmiento LA, Martínez JD. Coágulos intraluminales en postoperatorio de una hepaticoyeyunostomía: rara complicación para un rápido actuar. MedUNAB. 2020;23(2): 294-300. doi: $10.29375 / 01237047.3823$

\section{RESUMEN}

Introducción. La obstrucción intestinal por coágulo intraluminal es una complicación posquirúrgica poco frecuente reportada en la literatura. Reportamos el caso de un paciente con obstrucción intestinal por coágulos intraluminales posquirúrgicos de una hepaticoyeyunostomía con Y de Roux. Caso clínico: Paciente masculino de 53 años de edad, se presenta con cuadro de colangitis recurrente, secundaria a estenosis benigna de la vía biliar post colecistectomía laparoscópica, realizada hace 2 años. En su manejo con endoprótesis por colangiopancreatografía retrógrada 


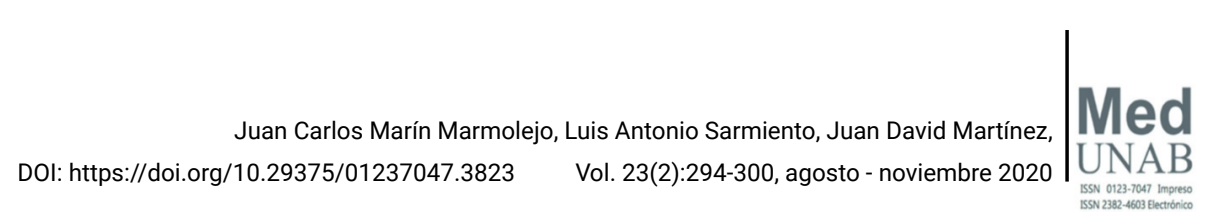

endoscópica (CPRE) no mejoró de sus episodios de colangitis, por lo que se consideró el paso a una reconstrucción tipo hepaticoyeyunostomía. En el postoperatorio temprano, presentó signos de obstrucción intestinal. La tomografía computarizada (TC) abdominal contrastada demostró líquido libre y signos de obstrucción. Es llevado a cirugía de urgencia, encontrándose la anastomosis en Y de Roux obstruida por gran coágulo intraluminal, requiriendo enterotomía para su extracción. Discusión: La obstrucción intestinal postoperatoria secundaria a un coágulo intraluminal es inusual, se debe sospechar en paciente con síntomas de obstrucción intestinal. El método ideal para el diagnóstico es la tomografía y el tratamiento es con relaparotomía para extraer los coágulos. Es importante el conocimiento de esta entidad para cualquier médico, ya que es una rara complicación en la que se necesita una buena sospecha diagnóstica y un tratamiento oportuno.

Palabras claves:

Trombosis, Intestino Delgado, Obstrucción Intestinal, Gastrectomía, Periodo Posoperatorio.

\begin{abstract}
Introduction. An intestinal obstruction due to intraluminal blood clots is a postoperative complication that is infrequently reported in literature. We reported the case of a patient with an intestinal obstruction due to postoperative intraluminal blood clots from a Roux-en-Y hepaticojejunostomy. Clinical Case: A 53-year-old male patient appeared with recurring cholangitis secondary to a benign stenosis of the bile duct after a laparoscopic cholecystectomy performed 2 years prior. Treatment with an endoprosthesis by endoscopic retrograde cholangiopancreatography (ERCP) did not improve cholangitis episodes, for which reason transition to reconstruction by hepaticojejunostomy was considered. He showed signs of intestinal obstruction during early postoperative care. The computed tomography (CT) scan of the abdomen with contrast showed free fluid and signs of obstruction. The patient was taken to an emergency surgery, where the Roux-en-Y anastomosis was found to be obstructed by a large intraluminal blood clot. It required an enterotomy to be extracted. Discussion: A postoperative intestinal obstruction secondary to intraluminal blood clots is unusual, but must be suspected in patients with symptoms of intestinal obstruction. The ideal method for diagnosis is by tomography and treatment involves a relaparotomy to extract the blood clots. It is important for doctors to have knowledge of this condition, since it is a rare complication that requires good diagnostic insight and timely treatment.
\end{abstract}

Keywords:

Thrombosis, Small Intestine, Intestinal Obstruction, Gastrectomy, Postoperative Period.

\title{
RESUMO
}

Introdução. Obstrução intestinal por coágulo intraluminal é uma complicação pós-cirúrgica rara relatada na literatura. Relatamos o caso de um paciente com obstrução intestinal por coágulo intraluminal pós-cirúrgicos de uma hepático-jejunostomia em Y-de- Roux. Caso clínico: Paciente do sexo masculino, 53 anos, apresentou colangite recorrente, secundária a estenose benigna do ducto biliar após colecistectomia laparoscópica, realizada 2 anos atrás. Em seu manejo com endoprótese por colangiopancreatografia endoscópica retrógrada (CPRE), seus episódios de colangite não melhoraram; portanto, foi considerado o passo para uma reconstrução do tipo hepático-jejunostomia. No pós-operatório inicial, apresentou sinais de obstrução intestinal. A tomografia computadorizada (TC) de abdome de contraste mostrou líquido livre e sinais de obstrução. O paciente foi levado para cirurgia de emergência, encontrando a anastomose em Y-de- Roux obstruída por um grande coágulo intraluminal, exigindo uma enterotomia para sua extração. Discussão: A obstrução intestinal pós-operatória secundária a um coágulo intraluminal é incomum, deve-se suspeitar de um paciente com sintomas de obstrução intestinal. O método ideal para o diagnóstico é a tomografia, e o tratamento é com relaparotomia para remover os coágulos. O conhecimento dessa entidade é importante para qualquer médico, pois é uma complicação rara que requer boa suspeita diagnóstica e tratamento imediato.

Palavras-chave:

Trombose; Intestino Delgado; Obstrução Intestinal; Gastrectomia; Período Pós-operatório. 


\section{Introducción}

La obstrucción intestinal por coágulo intraluminal, es una complicación posquirúrgica poco frecuente reportada en la literatura, entre sus causas está la Y de Roux, la cual se ha utilizado ampliamente en procedimientos como la gastrectomía para el cáncer, el bypass gástrico para la obesidad y en la hepaticoyeyunostomía o la coledocoyeyunostomía como una anastomosis de extremo a extremo (1). Las tasas de complicaciones para la hepaticoyeyunostomía en $\mathrm{Y}$ de Roux son relativamente bajas en grandes series $(2,3)$, pero son de importante consideración para el cirujano, ya que esta es una complicación en la que se requiere una pronta reparación.

No se conoce la prevalencia de obstrucción intestinal por coágulos postoperatorios en la hepaticoyeyunostomía en el mundo y en Colombia no se han reportado casos similares; sin embargo, la principal causa de obstrucciones intestinales por coágulos posquirúrgicos se presenta en la cirugía de bypass gástrico laparoscópico y tienen un tiempo promedio de presentación de 15 días, con un rango aproximado de 5 a 15 días (4). Las diferentes etiologías que generan una obstrucción intestinal después de una cirugía de bypass gástrico comprenden: incumplimiento de la dieta en un $46 \%$, edema anastomótico en un $23 \%$, estrechamiento de la yeyunostomía con un $23 \%$ y coágulos intraluminales en un $8 \%(4,5)$.

En la hepaticoyeyunostomía, las principales complicaciones son la obstrucción intestinal (adhesión e intususcepción), que representa el $4.5 \%$ de las complicaciones, y aproximadamente el $4 \%$ de los pacientes refieren pancreatitis o colangitis ascendente (3)

Esta es una entidad muy poco frecuente sin reportes a nivel nacional, probablemente por el subregistro; sin embargo, es de gran sospecha diagnóstica, ya que, en caso de presentarse esta complicación, es menester una rápida confirmación por imágenes (principalmente la tomografía) y un tratamiento oportuno. Como objetivo, la formación de coágulos intraluminales que causan obstrucción intestinal debe incluirse en el diagnóstico diferencial cuando se enfrentan a hallazgos clínicos similares en el período postoperatorio inmediato después de una hepaticoyeyunostomía, por lo que es importante, para el cirujano y el médico general, el conocimiento de esta complicación. Reportamos el caso de un paciente con obstrucción intestinal por hematoma intraluminal posquirúrgico de una hepaticoyeyunostomía con Y de Roux.

\section{Caso clínico}

Paciente masculino de 53 años de edad, con antecedentes de diabetes mellitus tipo 2, hipertensión arterial, esteatosis hepática grado III, insuficiencia renal aguda estadio V que requirió hemodiálisis. En el 2017 se le realizó colecistectomía abierta a causa de una coledocolitiasis, la cual no se pudo resolver por CPRE; secundario a esto, presenta colangitis recurrente, la cual es manejada un año después con endoprótesis por CPRE, presentando recurrencia en los episodios de colangitis, por lo cual no se encontró mejoría.

Se presenta con cuadro clínico de 10 días de evolución, consistente en dolor abdominal en epigastrio, prurito generalizado, fiebre de $38.5^{\circ}$, por lo que se considera diagnóstico de colangitis y se inicia manejo antibiótico. Al examen físico se encuentra ictérico, con dolor a la palpación abdominal y sin signos de irritación peritoneal. Se realiza colangioresonancia que evidencia dilatación severa de la vía biliar extrahepática y del conducto de Wirsung con presencia de cálculos en su interior; por lo cual, se decide realizar reconstrucción de la vía biliar. Se decide reconstruir la vía biliar con Y de Roux con anastomosis hepatoyeyunal término lateral transmesocólica, anastomosis intestinal latero lateral y se instala dren de nelaton subhepático, sin complicaciones intra quirúgicas, ni en el postoperatorio inmediato.

En el segundo día postoperatorio, presentó hematemesis en importante cantidad sin alteración de signos vitales, por lo que se decide suspender la enoxaparina y se inicia goteo de omeprazol. Posteriormente, la hemoglobina baja a $8 \mathrm{gr} / \mathrm{dl}$, por lo que se transfunden 2 unidades de concentrado globular. Al siguiente día, la hemoglobina sube a $9 \mathrm{gr} / \mathrm{dl}$ sin mejoría del cuadro.

El paciente continúa con dolor abdominal y empeoramiento de su estado general, se realiza tomografía computarizada (TC) abdominal contrastada que evidencia signos de obstrucción intestinal y líquido libre, con ocupación del intestino proximal, por lo cual es llevado a relaparotomía. Se encuentra obstrucción de intestino delgado proximal secundaria a taponamiento por abundantes coágulos a nivel de la Y de Roux, se hace enterotomía distal y se extraen $250 \mathrm{cc}$ de coágulos intraluminales (Figura 1), se refuerza la Y de Roux en su parte anterior, se instala un dren anterior cercano al píloro en caso de resangrado, se extrae el dren al flanco derecho, se deja bolsa de 1 litro y se traslada a UCI. El paciente evoluciona hacia la mejoría, por lo que se traslada a hospitalización a los 3 días y posteriormente es dado de alta a los 7 días sin nuevas complicaciones y sin nuevas recurrencias de colangitis. 
Figura 1. A: hallazgo de hematoma intraluminal en la Y de Roux, B: Enterotomía para extracción de coágulos, C: inicio de extracción de coágulos intraluminales, D: Finalización de la extracción de coágulos intraluminales.
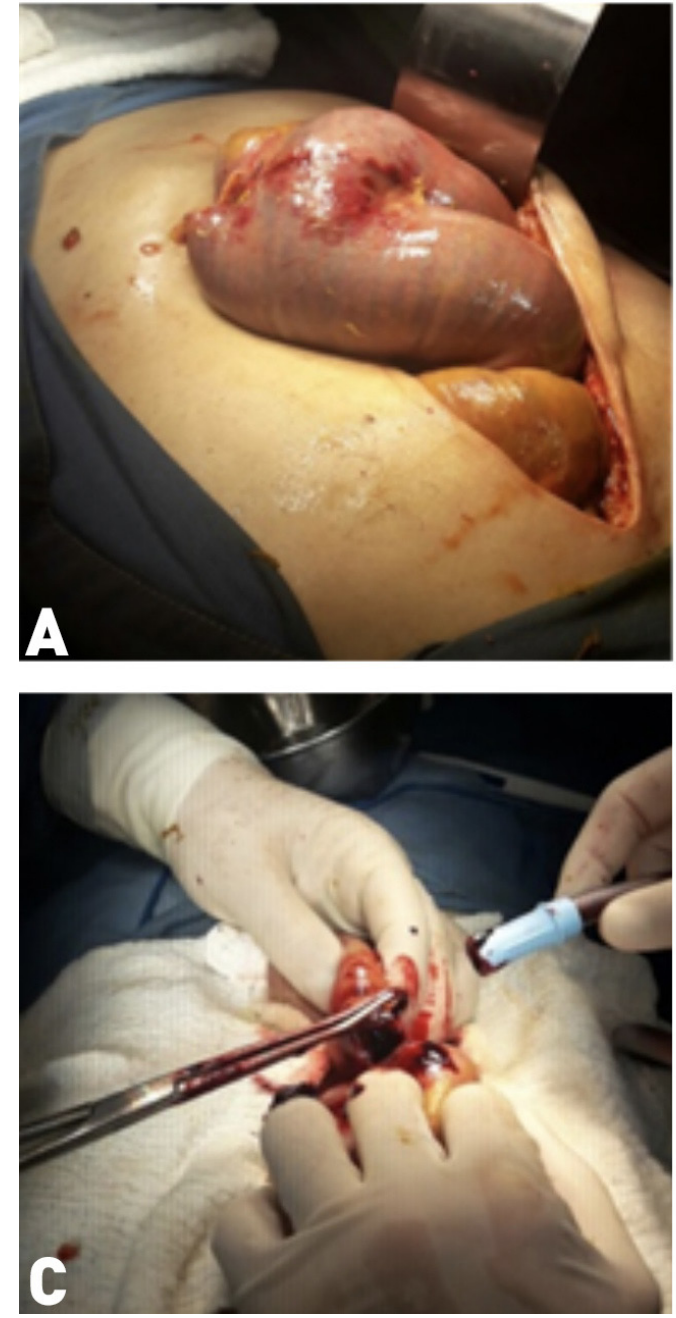

Fuente: Historia clínica del paciente

\section{Discusión}

La obstrucción intestinal postoperatoria secundaria a coágulos intraluminales es inusual (5) debe sospechar el cuadro clínicamente, ante síntomas de obstrucción mecánica del intestino delgado que incluye dolor abdominal tipo cólico, vómitos y constipación. El paciente presentaba claros síntomas de dolor abdominal tipo cólico y hematemesis proveniente del intestino proximal. Cuando se presenta obstrucción de asa cerrada y fiebre gangrenosa, se puede agregar sensibilidad abdominal y leucocitosis (6).

Cuando se presenta obstrucción intestinal por coágulos intraluminales, la hematemesis es un signo cardinal; además, es importante consultar regularmente los
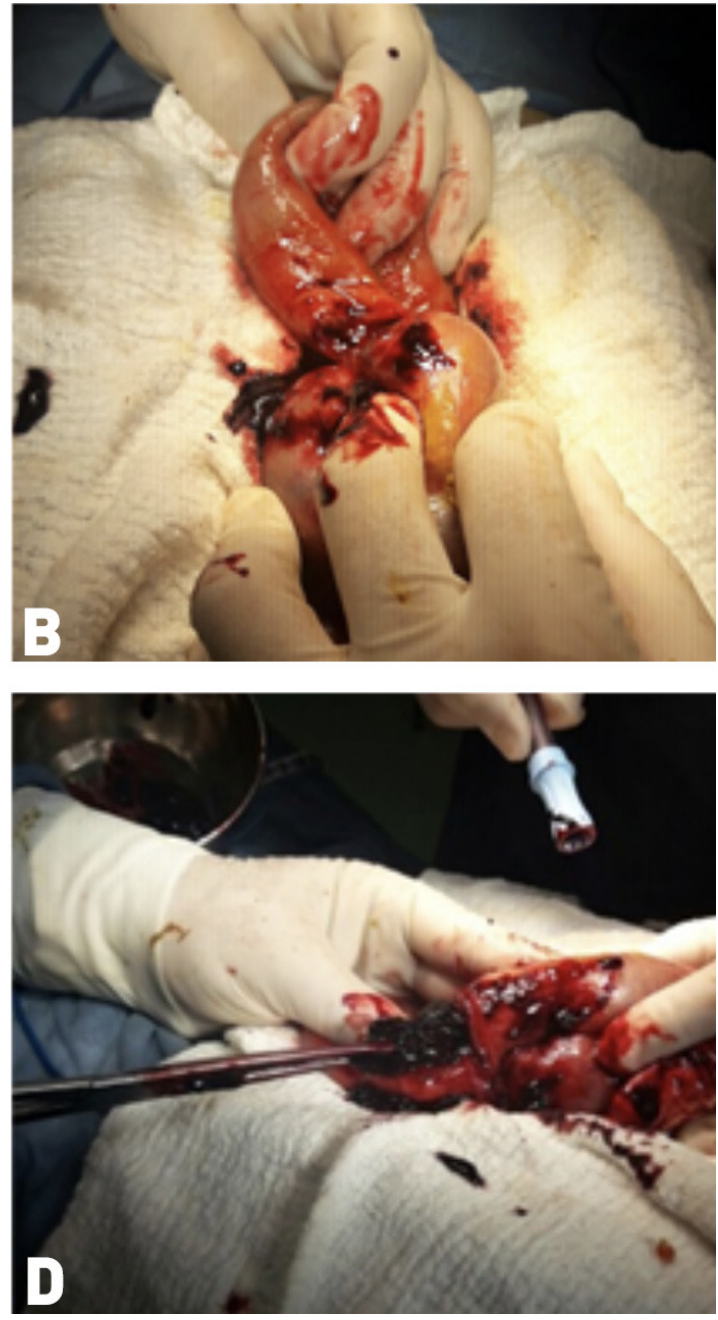

niveles de hemoglobina, ya que se genera una anemia por el sangrado intestinal, la cual se debe reponer con hemoconcentrados en caso de ser necesario, como se hizo en el paciente.

El uso de la tomografía computarizada para pacientes con esta presentación clínica ha sido el método diagnóstico de elección (7); además, la tomografía computarizada puede proporcionar información importante sobre la causa y la posición de la obstrucción y descartar otras posibles complicaciones postoperatorias tempranas (8).

La obstrucción resultante de la formación de coágulos sanguíneos intraluminales en el intestino delgado es causada por una hemostasia inadecuada en la anastomosis con grapas, una inadecuada verificación 
de las anastomosis o como causa de una enfermedad preexistente sin diagnóstico, como podría ser una alteración en la coagulación y rara vez se ve en el postoperatorio inmediato (9). En este caso, se verificó la adecuada hemostasia y se verificó la anastomosis. En cuanto a las comorbilidades, en la esteatosis hepática grado 3 que presentaba el paciente aún no se presenta disfunción del hígado que pueda explicar algún déficit en los factores de la coagulación; sin embargo, la insuficiencia renal crónica del paciente, por la cual ha requerido diálisis, puede ser causa de disfunción en la coagulación, que pudo contribuir a la complicación presentada.

Los principios de la técnica en la hepaticoyeyunostomía que minimizan las posibilidades de complicaciones comprenden la poca tensión, la anastomosis debe comenzar por ser lo más grande posible y debe haber una aposición completa del revestimiento del conducto biliar a la mucosa yeyunal.

Otra causa importante de obstrucción como diagnóstico diferencial es el hematoma intramural, el cual presenta síntomas semejantes con un tiempo postquirúrgico parecido al de los coágulos intraluminales; el primer informe de hematoma intramural en el hombre fue en 1838 por McLaughlan (10).

Se han descrito casos de obstrucción intestinal por hematoma intramural y coágulos intraluminales principalmente en traumas $(11,12)$, bypass gástrico laparoscópico con Y-de- Roux (8) (13-17), obstrucción espontánea como en el caso de un niño de 8 años (18), hemofilias (19), tratamientos anticoagulantes (20-24) y como complicación de la endoscopia (25). En la Tabla 1 se describen los casos de coágulos intraluminales con sus causas.

Tabla 1. Causas de coágulos intraluminales.

\section{COÁGULOS INTRALUMINALES}

\begin{tabular}{ccccccc}
\hline Año & Sexo & $\begin{array}{c}\text { Edad del } \\
\text { paciente }\end{array}$ & Lugar de obstrucción & Causa & Tratamiento & Referencia \\
\hline $\mathbf{2 0 1 7}$ & F & 29 & Yeyuno-yeyunostomía & Bypass gástrico & $\begin{array}{c}\text { Laparoscopia } \\
\text { y enterotomía }\end{array}$ & 9 \\
\hline $\mathbf{2 0 1 7}$ & M & 39 & Asa bilio pancreática & Bypass gástrico & $\begin{array}{c}\text { Laparoscopia } \\
\text { y enterotomía }\end{array}$ & 29 \\
$\mathbf{2 0 1 6}$ & M & 53 & Yeyuno-yeyunostomía & Bypass gástrico & $\begin{array}{c}\text { Laparoscopia } \\
\text { y enterotomía }\end{array}$ & 14 \\
\hline $\mathbf{2 0 1 5}$ & F & 20 & No especificado & Úlcera gástrica & $\begin{array}{c}\text { Lavado intestinal } \\
\text { con tubo }\end{array}$ & 30 \\
\hline $\mathbf{2 0 1 5}$ & M & 8 & Ileo distal & espontáneo & $\begin{array}{c}\text { Resección } \\
\text { segmentaria }\end{array}$ & 18 \\
\hline $\mathbf{2 0 1 4}$ & M & 71 & Yeyuno & $\begin{array}{c}\text { hepaticoyeyu- } \\
\text { nostomía }\end{array}$ & $\begin{array}{c}\text { Laparoscopia } \\
\text { y enterotomía }\end{array}$ & 1 \\
\hline $\mathbf{2 0 1 4}$ & F & 34 & Yeyuno-yeyunostomía & Bypass gástrico & $\begin{array}{c}\text { Laparoscopia } \\
\text { y enterotomía }\end{array}$ & 7 \\
\hline $\mathbf{2 0 1 2}$ & M & 68 & Duodeno proximal & Úlcera por & $\begin{array}{c}\text { Laparoscopia } \\
\text { y enterotomía }\end{array}$ & 31 \\
\hline $\mathbf{2 0 0 9}$ & F & 38 & Yeyuno-yeyunostomía & Bypass gástrico & $\begin{array}{c}\text { Laparoscopia } \\
\text { y enterotomía }\end{array}$ & 15 \\
\hline $\mathbf{2 0 0 5}$ & F & 35 & Yeyuno-yeyunostomía & Bypass gástrico & $\begin{array}{c}\text { Revisión de la } \\
\text { anastomosis }\end{array}$ & 17 \\
\hline $\mathbf{1 9 9 8}$ & M & 54 & Yeyuno-yeyunostomía & Gastrectomía & $\begin{array}{l}\text { Laparoscopia } \\
\text { y enterotomía }\end{array}$ & 32 \\
\hline
\end{tabular}

Fuente: Elaboración propias 
laparoscopic gastric bypass: case series and treatment algorithm. Surg Obes Relat Dis. 2009;5(2):203-207. https://doi.org/10.1016/j.soard.2008.10.003

5. Koppman JS, LiC, GandsasA. Smallbowel obstruction after laparoscopic Roux-En-Y gastric bypass: a review of 9,527 patients. J Am Coll Surg. 2008;206:571-84. https://doi.org/10.1016/j.jamcollsurg.2007.10.00

6. Pazouki A, Pakaneh M, Khalaj A, Tamannaie Z, Jangjoo A, ShapooriP, etal. Blood bezoarcausing obstruction after laparoscopic Roux-en-Y gastric bypass. Int J Surg Case Rep [Internet]. Surgical Associates Ltd. 2014;5(4):183-5. https://doi.org/10.1016/j.ijscr.2013.12.022

7. Abbas MA, Collins JM, Olden KW, et al. Spontaneous intra-mural small-bowel hematoma: clinical presentation and long-term outcome. Arch Surg. 2002;137:306-310. https://doi.org/10.1001/archsurg.137.3.306

8. Soricelli E, Facchiano E, Quartararo G, Beltrame B, Leuratti L, Lucchese M. Large Hemobezoar Causing Acute Small Bowel Obstruction After Roux-en-Y Gastric Bypass: Laparoscopic Management. Obes Surg. Obesity Surgery; 2017;27(7):1906-7. https:// doi.org/10.1007/s11695-017-2708-4

La obstrucción intestinal por coágulos intraluminales es una complicación de la hepaticoyeyunostomía con Y de Roux muy poco frecuente. Sin embargo, es importante para el médico conocer esta complicación del postoperatorio, ya que se necesita un diagnóstico oportuno basándose en síntomas como hematemesis y dolor abdominal intenso tipo cólico. Con lo cual, se debe confirmar el diagnóstico con una tomografía abdominal para ser llevado a cirugía para enterotomía con corrección de la anastomosis, en caso de necesitarse.

\section{Referencias}

1. Shah MM, Rather AA, Khan FA. Acute Bowel Obstruction After Hepaticojejunostomy Caused by Blood Clots. Journal of the society of laparoendoscopic surgeon. 2014;30(2):1-4. https://doi.org/10.4293/ CRSLS.2014.00188

2. Ohi R, Yaoita S, Kamiyama T, Ibrahim M, Hayashi Y, Chiba T. Surgical treatment of congenital dilatation of the bile duct with special reference to late complications after total excisional op- eration. J Pediatr Surg. 1990;25(1):613-617. https://doi. org/10.1016/0022-3468(90)90346-B

3. Yamataka A, Ohshiro K, Okada Y, et al. Complications after cyst excision with hepaticoenterostomy for choledochal cysts and their surgical management in children versus adults. J Pedi- atr Surg. 1997; 32(2):1097-1102. https://doi.org/10.1016/S00223468(97)90407-3

4. Lewis CE, Jensen C, Tejirian T, Dutson E, Mehran A. Early jejunojejunostomy obstruction after
9. Felsher J, Brodsky J, Brody F. Small bowel obstruction after laparoscopic Roux-en-Y gastric bypass. Surgery. 2003;134(3):501-505. https://doi.org/10.1067/S00396060(03)00251-4

10. McLaughlan J. Fatal false aneurysmal tumour occupying nearly the whole of the duodenum. Lancet 2. 1838:30(766). https://doi.org/10.1016/S01406736(02)95675-8

11. Homma Y, Mori K, Ohnishi Y, Fujioka K, Terada T, Sasaki A, et al. Ultrasound follow-up in a patient with intestinal obstruction due to post-traumatic intramural duodenal hematoma. J Med Ultrason (2001) [Internet]. 2016 Jul 18 [cited 2018 Apr 26];43(3):431-4. https:// doi.org/10.1007/s10396-016-0717-x

12. Chang C-W, Chen M-J, Shih S-C, Wang H-Y, Tsai C-H, Wang T-E, et al. Delayed duodenal intramural hematoma with obstruction. Am J Surg. 2010 Jun [cited 2018 Apr 26];199(6):e77-8. https://doi. org/10.1016/j.amjsurg.2009.07.046

13. Green J, Ikuine T, Hacker S, Urrego H, Tuggle K. Acute small bowel obstruction due to a large intraluminal blood clot after laparoscopic Roux-en-Y gastric bypass. J Surg Case Reports. 2016;2016(8):rjw143. https://doi.org/10.1093/jscr/rjw143

14. Peeters G, Gys T, Lafullarde T. Small Bowel Obstruction after Laparoscopic Roux-en-Y Gastric Bypass Caused by an Intraluminal Blood Clot. Obes Surg. 2009 Apr 6;19(4):521-3. https://doi.org/10.1007/ s11695-008-9575-y

15. Helling TS, Balliro JM, Nguyen NT, Longoria M, Wilson SE. The lethality of obstructing hematoma at the jejunojejunostomy following Roux-en-Y gastric 
bypass. Obes Surg. 2005;15(2):290-3. https:// doi.org/10.1381/0960892053268318

16. Awais O, Raftopoulos I, Luketich JD, Courcoulas A. Acute, complete proximal small bowel obstruction after laparoscopic gastric bypass due to intraluminal blood clot formation. Surg Obes Relat Dis. 2005;1(4):41822. https://doi.org/10.1016/j.soard.2005.04.004

17. Lim YJ, Nam SH, Kim SJ. Large intraluminal ileal hematoma presenting as small bowel obstruction in a child. Iran J Radiol. 2015 Apr 22;12(2):e8212. https:// doi.org/10.5812/iranjradiol.8212

18. Delibegovic M, Alispahic A, Gazija J, Mehmedovic $\mathrm{Z}$, Mehmedovic M. Intramural Haemorrhage and Haematoma as the Cause of Ileus of the Small Intestine in a Haemophiliac. Med Arch (Sarajevo, Bosnia Herzegovina). 2015 Jun;69(3):206-7.

19. Schroeder RM, Kohler JE, Mak GZ, Kandel JJ. Bowel obstruction from intramural hematoma in two children treated with low molecular weight heparin: Case report and review of the literature. J Pediatr Surg Case Reports [Internet]. 2014;2(10):483-5. https://doi. org/10.1016/j.epsc.2014.09.013

20. Zammit A, Marguerat DG, Caruana C. Anticoagulation-induced spontaneous intramural small bowel haematomas. BMJ Case Rep [Internet]. 2013 Jun 3;2013:bcr2013008831. https://doi. org/10.1136/bcr-2013-008831

21. Brar P, Singh I, Kaur S, Doley RP, Brar R, Sahni A, et al. Anticoagulant-induced intramural hematoma of the jejunum. Clin J Gastroenterol [Internet]. 2011 Dec 30 [cited 2018 Apr 26];4(6):387-90. https://doi. org/10.1007/s12328-011-0256-3

22. eldt BJ, Haringsma J, Florijn KW, Kuipers EJ. Coumarin-induced intramural hematoma of the duodenum: case report and review of the literature. Scand J Gastroenterol [Internet]. 2011 Mar 14 [cited 2018 Apr 26];46(3):376-9. https://doi.org/10.3109/00 365521.2010.531484

23. Altikaya N, Parlakgümüş A, Demır Ş, Alkan Ö, Yildirim T. Small bowel obstruction caused by intramural hematoma secondary to warfarin therapy: a report of two cases. Turk J Gastroenterol [Internet]. 2011 [cited 2018 Apr 26];22(2):199-202. https://doi. org/10.4318/tjg.2011.0192

24. Grasshof C, Wolf A, Neuwirth F, Posovszky C. Intramural duodenal haematoma after endoscopic biopsy: case report and review of the literature. Case Rep Gastroenterol [Internet]. 2012 Jan [cited 2018 Apr 26];6(1):5-14. https://doi.org/10.1159/000336022

25. Wassner JD, Yohai E, Heimlich HJ. Complications associated with the use of gastrointestinal stapling devices. Surgery. 1977;82(4):395-399.

26. Birns MT, Katon RM, Keller F. Intramural hematoma of the small intestine presenting with major upper gastrointestinal hemorrhage. Gastroenterology. 1979;77(2):1094-1100. $\quad$ https://doi.org/10.1016/ S0016-5085(79)80084-0

27. Eiland M, Han SY, Hicks GM. Intramural hemorrhage of the small intestine. JAMA. 1978;239(3):139-142.

28. Shimizu H, Maia M, Kroh M, Schauer PR, Brethauer SA. Surgical management of early small bowel obstruction after laparoscopic Roux-en-Y gastric bypass. Surg Obes Relat Dis [Internet]. 2013 Sep [cited 2018 Apr 26];9(5):718-24. https://doi.org/10.1016/j. soard.2012.05.009

29. Ruiz-Tovar J. Hemobezoar causante de obstrucción intestinal a nivel del pie de asa durante el postoperatorio de un Bypass Gástrico en Y de Roux. BMI | Bariátrica Metabólica Ibero-Americana [Internet]. 2017 Sep 29 [cited 2018 Apr 26];7(3):1859-1961.

30. Mizumura N, Imagawa A, Kawasaki M, Okumura S, Toyoda S, Ogawa M. Small Bowel Obstruction Caused by Blood Clots: A Rare Complication of Peptic Ulcer. J Med Cases [Internet]. 2015;6(10):4779. https://doi.org/10.14740/jmc2298w

31. Siddiky A, Gupta P. Proximal small bowel obstruction caused by a massive intraluminal thrombus from a stress ulcer. J Surg case reports [Internet]. 2012 Jan 1 [cited 2018 Apr 26];2012(1):6. https://doi. org $/ 10.1093 / \mathrm{jscr} / 2012.1 .6$

32. Onda M, Urazumi K, Abe R, Matsuo K. Obstructive Ileus caused by blood clot after emergency total gastrectomy in a patient with hemophilia A: report of a case. Surg Today [Internet]. 1998 [cited 2018 Apr 26];28(12):1266-9. https://doi.org/10.1007/ $\underline{\mathrm{BF} 02482812}$ 